\title{
Bounds on the Throughput Gain of Network Coding in Unicast and Multicast Wireless Networks
}

\author{
Junning Liu, Dennis Goeckel, and Don Towsley
}

\begin{abstract}
Gupta and Kumar established that the per node throughput of ad hoc networks with multi-pair unicast traffic scales with an increasing number of nodes $n$ as $\lambda(n)=$ $\Theta(1 / \sqrt{n \log n})$, thus indicating that performance does not scale well. However, Gupta and Kumar did not consider network coding and wireless broadcasting, which recent works suggest have the potential to significantly improve throughput. Here, we establish bounds on the improvement provided by such techniques. For random networks of any dimension under either the protocol or physical model that were introduced by Gupta and Kumar, we show that network coding and broadcasting lead to at most a constant factor improvement in per node throughput. For the protocol model, we provide bounds on this factor. We also establish bounds on the throughput benefit of network coding and broadcasting for multiple source multicast in random networks. Finally, for an arbitrary network deployment, we show that the coding benefit ratio is at most $O(\log n)$ for both the protocol and physical communication models. These results give guidance on the application space of network coding, and, more generally, indicate the difficulty in improving the scaling behavior of wireless networks without modification of the physical layer.
\end{abstract}

Keywords: Ad Hoc Networks, Scaling Laws, Network Coding, Wireless Broadcasting

\section{INTRODUCTION}

$\mathbf{M}$ ULTI-HOP wireless networks have been intensively studied in recent years for both commercial and government applications. Such networks, static or mobile, have the potential to serve as either a self-contained network that provides communication without the presence of an established infrastructure, or as an ubiquitous bridge between end users and the high speed wired infrastructure. Hence, issues of the connectivity and capacity of such networks are of interest.

One major concern with wireless networks is scalability. Under a traditional communication model without network

Manuscript received 1 August 2008; revised 20 February 2009. This research is continuing through participation in the International Technology Alliance sponsored by the U.S. Army Research Laboratory and the U.K. Ministry of Defence under Agreement Number W911NF-06-3-0001. This work is also performed under a subcontract to BAE Systems for the Defense Advanced Research Project Agency and the Space and Naval Warfare Systems Center, San Diego, under Contract no. N66001-06-C-2020. Part of the work is also supported by the National Science Foundation under ECS-0300130, CCF-0430892, CNS-0721861, and NSF equipment grant NSF EIA-0080119. Any opinions, findings, and conclusions or recommendations expressed in this material are those of the authors and do not necessarily reflect the views or policies of the funding agencies. Preliminary versions of this work appeared at the 2006 Military Communications Conference, Washington DC, USA, and at 2007 IEEE InfoCom, Anchorage, Alaska, USA.

Junning Liu and Don Towslety are with the Dept. of Computer Science, University of Massachusetts, Amherst, MA (e-mail: \{liujn,towsley\}@cs.umass.edu).

Dennis Goeckel is with the Dept. of Electrical and Computer Engineering, University of Massachusetts, Amherst, MA (e-mail: goeckel@ecs.umass.edu).

Digital Object Identifier 10.1109/JSAC.2009.0906xx. coding, [1] studies the network capacity for randomly deployed wireless networks. For multi-pair unicast traffic, which means there are multiple unicast source destination pairs that try to deliver the same data rate across all pairs, [1] shows that the per node throughputs of such random networks scales as $\lambda(n)=\Theta\left(\frac{1}{\sqrt{n \log n}}\right)$, where $n$ is the total number of nodes in the network and each node sends to a randomly chosen destination at a rate of $\lambda(n)$. Thus, as the total number of nodes increases, the pairwise throughput does not scale well. To increase throughput, mobility [2] or hierarchical cooperation involving physical layer modification [3] can achieve a constant throughput at a cost of unbounded delay. Liu et. al. [4] studies hybrid networks with infrastructure support and finds that the infrastructure has to be sufficiently dense to make a difference in the asymptotic order of the throughput.

Another thread of work is to study in-network computation. The work by Ahlswede, Cai, Li and Yeoung [5] introduces the concept of network coding (NC), and there has been tremendous interest in applying network coding in both wired [6] and wireless networks [7] [8] [9]. For the wired case, the benefit of network coding in terms of throughput and capacity is often limited. Specifically, for networks with bidirectional links that can be modeled as an arbitrary undirected graph, [6] shows that the throughput improvement is upper bounded by a factor of two for the single source multicast case, and upper bounded by one (no benefit) for the single source unicast or broadcast case. In addition, it is conjectured that there is no throughput benefit for the multi-pair unicast case; this is called the Li\&Li conjecture, which is still open with no counter-examples found yet.

Wireless networks cannot be modeled as undirected graphs. Network coding, combined with wireless broadcasting, can potentially improve the performance on throughput [8], [9], energy efficiency and congestion control [7], [8]. In addition, recent work by Katti et al. [10] demonstrates the potential throughput benefit of applying network coding to wireless networks through constructive examples and experiments. Since network coding was not taken into consideration in Gupta\&Kumar's original work [1] and the related works that followed, an interesting question raised after [10] is how much throughput benefit can it provide in wireless networks. Answering this question will help us to better understand not only the benefit and limitations of network coding on the capacity of wireless networks and networks in general, but also the degree of scalability of random wireless networks, thus providing design guidelines for the coverage ratio between wireless mesh net and the infrastructure wired net in hybrid networks. 


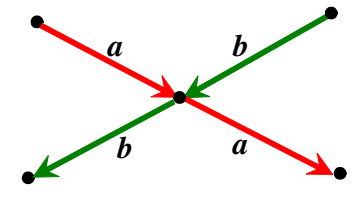

Without coding/broadcasting 4 transmissions

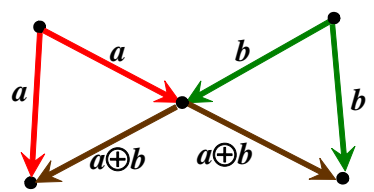

With coding/broadcasting 3 transmissions
Fig. 1. An example demonstrating the benefit of Katti etc. [10]'s opportunistic coding scheme

The idea of [10] is to use network coding to combine (code together) data from intersecting flows, and then broadcast this coded information simultaneously to multiple next hop receivers. Each next hop relay node for a given flow decodes that flow's traffic based on all of the broadcasts that it has received as well as on local information (source data generated locally). In this way a node can potentially deliver multiple data flows to multiple neighbors with a single broadcast transmission. An example of this is shown in Fig. 1. Thus, intuitively it appears that there could be a throughput benefit ratio proportional to the expected number of neighbors, $\Theta(\log n)$ in the case of uniformly random deployed networks. However, we demonstrate in this paper that such an improvement is not possible for any network coding scheme under either the protocol or physical models of Gupta and Kumar; in fact, only a constant improvement in throughput can be achieved. We further provide bounds on the scalar throughput benefit ratio under the protocol model. A summary of these results is provided in Table 1.

Next, our attention is turned to the multiple source multicast problem in large random networks. For a constant number of destination nodes $n_{d}$ per each of the $n_{s}=n$ source nodes, we establish that the throughput benefit of network coding and broadcasting is again bounded by a scalar. When the number of source nodes is $n_{s}=n^{\epsilon}$ and the number of destination nodes is $n_{d}=n^{1-\epsilon}$, the throughput improvement of network coding and broadcasting is upper bounded by $\sqrt{\log n \sqrt{n^{1-\epsilon}}}$.

Finally, in addition to asymptotic results that focus on a randomly deployed network with a large number of nodes, we also consider a wireless network with an arbitrary nodes deployment and an arbitrary subset of the nodes as sources and destinations. Under some stationarity assumptions on the network coding solution, we show that the coding benefit ratio is at most a factor of $O(\log n)$ in this case for one-dimensional, two-dimensional, or three-dimensional spaces, where $n$ is the total number of sources and destinations.

The remainder of this paper is organized as follows. Section II reviews related work. Section III introduces the physical and protocol models for randomly deployed networks. Section IV considers the throughput gain provided by network coding and broadcasting for multi-pair unicast traffic by establishing the unicast results in Table 1, whereas Section V considers the multi-pair multicast case. Section VI considers the gain in arbitrary networks. Section VII provides the conclusions.

\section{RELATED WORK}

Here, we briefly review other works that focus on the throughput benefit provided by network coding (NC) [5] in various scenarios. For the wired case, the benefit of network coding in terms of throughput and capacity is often limited. Specifically, for networks with bidirectional links that can be modeled as an arbitrary undirected graph, [6] shows that the throughput improvement is upper bounded by a factor of two for the single multicast case, and nonexistent for the single unicast or broadcast case.

Katti et al. [10] demonstrates the potential throughput benefit of applying network coding to wireless networks through constructive examples and experiments. Alimi et al. [11] demonstrates by simulations that further throughput gain is possible through mixed network layer coding and physical layer superposition coding. Another work by Le et al. [12] studies [10]'s type of point-to-point XOR coding based on random access physical layers, where point-to-point coding means that packets are decoded at each hop before being forwarded on. [11] provides upper bounds on the maximum number of packets that can be encoded together.

Under a different definition of the model for an arbitrary network than considered here, the work of [13] shows that for single source multicast, coding provides at most a constant factor improvement on throughput. We hasten to note the distinction of the arbitrary network model of [13] from that of Section VI of this paper; in particular, [13] establishes the constant improvement factor of network coding on transport capacity when the transport capacity of each of the network coding and the flow schemes is maximized over all possible sets of source-destination specifications. In contrast, the result in Section VI applies to a single given (but arbitrary) wireless network deployment and set of source-destination specifications. In [13], a corollary to their main result and Gupta\&Kumar's former result [1], Corollary 3, shows that the throughput improvement provided by network coding in the random network multi-pair unicast case is bounded by a constant, thus providing an alternate proof for one of our main results that was originally established in [14].

Finally, there are works subsequent to the preliminary conference versions of this paper that extend our cut technique of [14], [15] to other communication models such as multipacket reception (MPR), physical layer network coding (PNC) and analog network coding (ANC) [16], [17], [18], [19], [20].

\section{MODEL FORMULATION}

We consider the network model of Gupta\&Kumar [1], where $n$ nodes are randomly located, i.e., independently and uniformly distributed, in a fixed size region. We do not limit the shape of the region or its dimension. However, for simplicity of presentation, we derive our results based on a unit square.

There are $n$ source-destination pairs in the network. Each node $i$ in the network is a data source that needs to route its data through multi-hop wireless communications to a destination node that is independently and uniformly randomly chosen. The same protocol and physical communication models are employed as in Gupta\&Kumar [1]. 


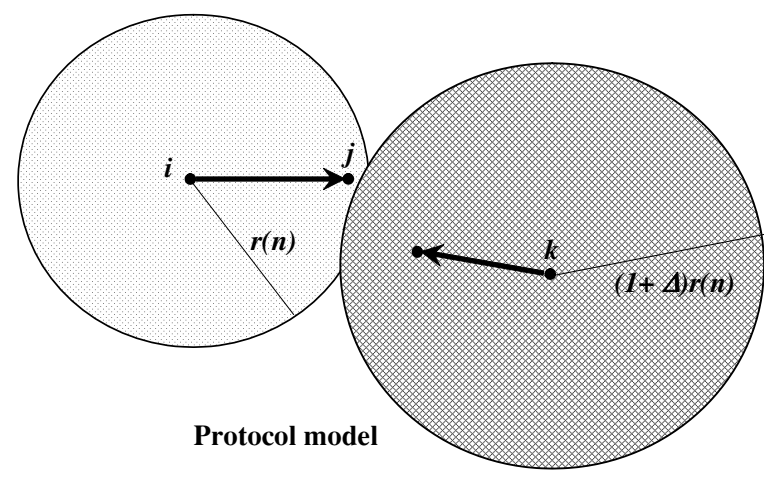

Fig. 2. The protocol communication model

For the protocol model (see Fig. 2), a transmission from node $i$ to $j$ is successful if and only if the distance between them satisfies $\left|X_{i}-X_{j}\right| \leq r(n)$ and any other simultaneously transmitting node $k$ satisfies $\left|X_{k}-X_{j}\right| \geq(1+\Delta) r(n)$. Here, $X_{i}$ is node $i$ 's location, $r(n)$ is the transmission radius and $\Delta>0$ ensures a safety zone that limits the interference; in particular, $\Delta$ is a constant that depends on the properties of the wireless medium. In addition, there is a finite bandwidth limit of $W$ bits/sec for each transmission. In order to ensure connectivity, the fixed transmission radius for the protocol model needs to be at least $r(n)=\Theta(\sqrt{\log n} / \sqrt{n})$ [1].

In the physical communication model [1], each transmission has a fixed power $P$, and a transmission from node $i$ to $j$ is successful if the signal to interference plus noise ratio is above a threshold:

$$
\frac{P / r_{i, j}^{\gamma}}{N+\Sigma_{k \in K} P / r_{k, j}^{\gamma}} \geq \beta
$$

where $K$ is the node set of all other nodes that are simultaneously transmitting, $N$ is the ambient noise power level and $\beta>0$ is the threshold.

The protocol model is a simple abstraction of the real situation that provides a nice basis for analysis yet captures the core interference effect of the real network, especially for asymptotic analysis. The protocol model yields same order throughput results as the physical model, which is a much more realistic model that models the signal decaying and interfering effect in wireless transmissions.

As in Gupta\&Kumar [1], attention here is focused on the data rate at which each node can send to its destination node. A throughput $\lambda(n)$ (bits/sec) is feasible if there exists a scheme that achieves $\lambda(n)$ on average. The throughput capacity of such a random network is defined as the maximum throughput that is feasible with high probability.

Here, transmission schemes correspond to the same type of "spatial and temporal scheduling schemes that operate the network in a multi-hop fashion and buffers at intermediate nodes when awaiting transmissions" as in [1]. Two types of schemes are considered: a flow scheme and a coding scheme. A flow scheme is a non-coding scheme where data are routed as commodity flows (duplication, forwarding, but no coding) and thus the broadcast nature of the wireless medium is not helpful for the flow scheme for the unicast task. Gupta\&Kumar [1] focus on the throughput of flow schemes. A coding scheme is one that allows all of the operations in a flow scheme, along with allowing messages received at each node to be decoded/recoded; in other words, intermediate nodes can send the results obtained from applying arbitrary functions to all previously received bits and its own source data as long as each destination node is able to decode the data intended for it from all of its received bits and local data. Thus all possible benefits of combining network coding and wireless broadcasting as demonstrated in Katti etc. [10] are incorporated in the considered coding schemes. The throughput capacity is denoted as $\lambda_{F}(n)$ for flow schemes and $\lambda_{C}(n)$ for coding schemes. The throughput benefit ratio of the coding scheme is denoted as $\alpha(n)=\lambda_{F}(n) / \lambda_{C}(n)$. As in Gupta\&Kumar [1], all packets are independent of each other whether they are from different sources or the same source.

\section{Throughput Benefit of CODing SCHEMES FOR Multi-Pair UnicAst TRAFFiC}

In this section, we show that under either the protocol or physical model, coding schemes provide at most a constant factor improvement in throughput over flow schemes. In other words, there exists some constant $c$ (i.e. not dependent on $n$ ) such that $\alpha(n) \leq c$. We also derive upper bounds for the constant factor in the protocol model case. Although our bounds are most conclusive in the one-dimensional case, throughout this section we focus on the two-dimensional case of most general interest, referring the interested reader to [15] and [21] for the proofs and discussion of the results in Table 1 in other numbers of dimensions.

\section{A. Sparsity Cut for a Random Network}

In general, a cut $\Gamma$ is defined as a partition of the nodes in a graph, the cut capacity is the sum of the links' bandwidths crossing the cut, and the sparsity cut is a cut where the cut capacity divided by the traffic demand is the minimum over all cuts. Since the network studied here is a random deployed network embedded in an Euclidean space and transmissions are between neighboring nodes, attention can be focused on a narrow class of cuts that are induced by a line segment that cuts the region into two regions. The cut length $l_{\Gamma}$ is defined as the length of the cut line segment. The cut lines that we consider have zero width measure such that no nodes lie on them. Denote the two subregions divided by the cut as $\Gamma_{1}$ and $\Gamma_{2}$. A sparsity cut for a random network is defined as a cut induced by the line segment with the minimum length that separates the region into two equal area subregions. For the square deployment region illustrated in Fig. 3, the line segment $A B$ induces a sparsity cut $\Gamma_{A B}$. Since nodes are uniformly randomly deployed in a random network, such a sparsity cut captures the traffic bottleneck of these random networks on average. The cut capacity is defined as $\left(\Lambda_{\Gamma_{1,2}}, \Lambda_{\Gamma_{2,1}}\right)$ where $\Lambda_{\Gamma_{1,2}}$ equals the transmission bandwidth $W$ times the maximum possible number of simultaneous transmissions (broadcast or non-broadcast) across the cut from $\Gamma_{1}$ to $\Gamma_{2}$; and $\Lambda_{\Gamma_{2,1}}$ equals the same quantity from $\Gamma_{2}$ to $\Gamma_{1}$. This cut capacity constrains the information rate that the nodes from one side of the cut as a whole can deliver to the nodes at the other side as a whole. The number of sources in $\Gamma_{1}$ whose destinations are in $\Gamma_{2}$ is denoted as $n_{\Gamma_{1,2}}$. 


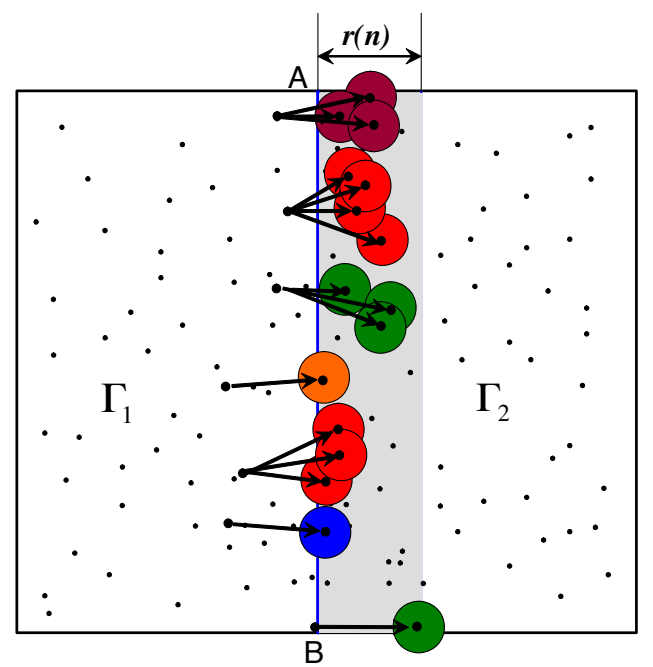

Fig. 3. Cut Capacity in 2D

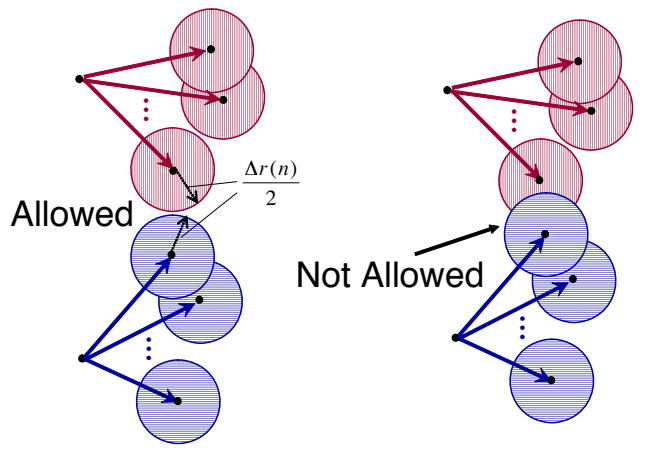

Fig. 4. Interference of coding schemes in 2D

\section{B. Throughput Order Improvement of the Coding Scheme in the Protocol Model}

The cut capacity is bounded by deriving an upper bound on the maximum number of simultaneous transmissions across the cut. It is easy to see that all of the direct receivers of transmissions across a cut $\Gamma$ in one direction lie in the shaded rectangle region with area $l_{\Gamma} \times r(n)$ as shown in Fig. 3. In [1], disks of radius $\Delta r(n) / 2$ centered at each receiver are disjoint; otherwise, some sender is within $(1+\Delta) r(n)$ of some other sender's receiver. However, [1] does not exploit broadcast transmissions while a coding scheme does. As shown in Fig. 4, with the consideration of broadcast and network coding, observe that while disks centered at receivers of the same sender (broadcast transmission) could overlap, disks centered at receivers of different senders are still disjoint. In other words, we make the following observation:

Observation 1: The union of disks (with radius $\Delta r(n) / 2$ ) centered at the receivers of one transmission should be disjoint from the union of disks centered at the receivers of another transmission.

Lemma 1: The capacity of a cut $\Gamma$ for a $2 D$ region has an upper bound of $\frac{c_{\Delta} l_{\Gamma} W}{r(n)}$ where $c_{\Delta}=\max \left\{\frac{16}{\pi \Delta^{2}}, \frac{\sqrt{3}}{\Delta}\right\}$

Proof: When $\Delta<2$, Observation 1 means each transmission across the cut consumes at least an area of $\frac{1}{4} \pi\left(\frac{\Delta r(n)}{2}\right)^{2}$ of the shaded region in Fig. 3, with the minimum achieved when a receiver lies in the corner of the shaded region. Thus,

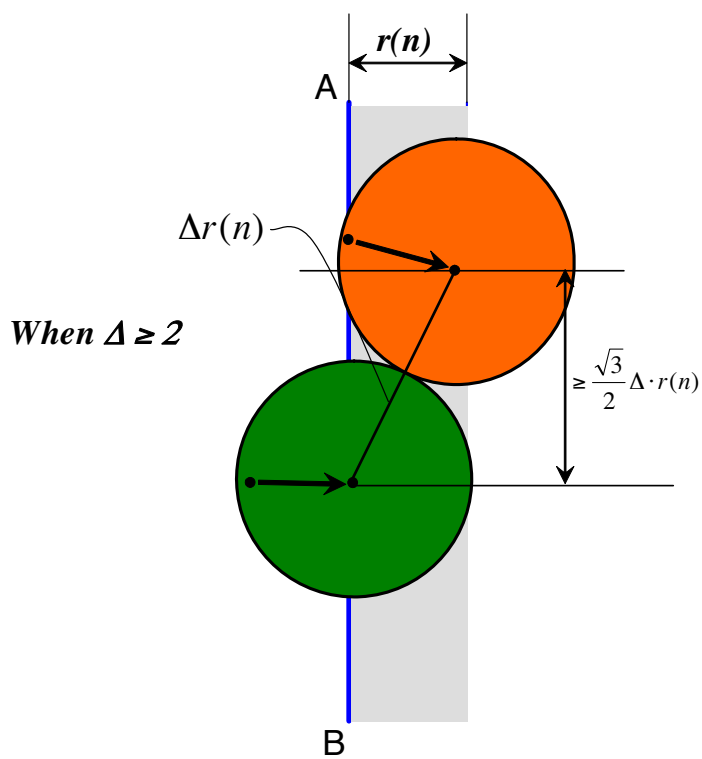

Fig. 5. $2 D$ Cut capacity: $\Delta \geq 2$ case

the maximum number of simultaneous transmissions across the cut is upper bounded by the area of the shaded region divided by $\frac{1}{4} \pi\left(\frac{\Delta r(n)}{2}\right)^{2}$, which is $\frac{16 l_{\Gamma}}{\pi \Delta^{2} r(n)}$.

When $\Delta \geq 2$, as shown in Fig. 5, any two receivers of two different transmissions require a $\frac{\sqrt{3}}{2} \Delta r(n)$ difference in their coordinates along the cut line. Thus, there can be at most $\frac{l_{\Gamma}}{\sqrt{3} \Delta r(n) / 2}+1 \leq \frac{\sqrt{3} l_{\Gamma}}{\Delta r(n)}$ simultaneous transmissions across the cut.

Since each transmission is able to send $W$ bits/sec, combining the two cases above, the cut capacity is upper bounded by $\Lambda_{\Gamma_{1,2}} \leq \frac{c_{\Delta} l_{\Gamma} W}{r(n)}$ and $\Lambda_{\Gamma_{2,1}} \leq \frac{c_{\Delta} l_{\Gamma} W}{r(n)}$, where $c_{\Delta}=$ $\max \left\{\frac{16}{\pi \Delta^{2}}, \frac{\sqrt{3}}{\Delta}\right\}$.

Corollary 1: The sparsity cut capacity of a $2 D$ random network has an upper bound of $c_{\Delta} W / r(n)$.

Proof: Regardless of the shape of the unit area region, the sparsity cut must have a length $l_{\Gamma} \leq 1$, as follows. Cuts that divide the region into two equal parts will pass through the centroid of the region. Hence, if the sparsity cut has length greater than one, all cuts across the centroid have a length larger than 1 and the area of the region will be bigger than 1 , which contradicts the unit area assumption. Hence, from Lemma 1, we derive the corollary.

Next we derive an upper bound for the throughput of all coding schemes in a $2 D$ random network.

Theorem 1: The throughput of coding schemes in a $2 D$ random network is upper bounded by $\Theta\left(\frac{W}{n r(n)}\right)=\Theta\left(\frac{W}{\sqrt{n \log n}}\right)$

Proof: Assume the coding throughput of the $n$ node random network is $\lambda_{C}(n)$. Then, by its definition, with high probability (w.h.p.) there exists some coding scheme that, for some $T<\infty$, during each time interval $[(i-1) T, i T]$ (in seconds), every node can send $T \lambda_{C}(n)$ bits of information to its corresponding destination node. For a sparsity cut $\Gamma_{A B}$ in the middle, by a Chernoff bound [22] argument, we have that w.h.p. there are $\Theta(n)$ pairs of source-destination nodes that need to cross $\Gamma_{A B}$ in one direction, i.e., $n_{\Gamma_{1,2}}=n_{\Gamma_{2,1}}=\Theta(n)$ w.h.p.. Now we view all of the nodes lying on the right side of $A B$ as a super node, and treat all of the distinct messages 
it receives from the left side of $A B$ within the time interval $[(i-1) T, i T]$ as a single 'meta' message $M$. We denote the number of bits of $M$ as $B_{M}$. According to the definition of our coding scheme, this meta message $M$ can be arbitrarily coded but with only one coding constraint: by Shannon's data compression theorem [23], in order for the right side destination nodes to decode the original data from the left side sources which are independent of each other, $M$ has to satisfy $B_{M} \geq T n_{\Gamma_{1,2}} \lambda_{C}(n)$ or $B_{M} \geq T \Theta(n) \lambda_{C}(n)$ w.h.p..

At the same time, we derive an upper bound on $B_{M}$ with the support of Corollary 1. A broadcast transmission across the cut to multiple receivers delivers identical information to the receivers; however, by the definition of $M$, the identical messages will only be counted once in $M$ for one of the receivers. Also by the definition of cut capacity and Corollary 1 , we get $B_{M} \leq c_{\Delta} W T / r(n)$. Combined with the coding constraint above, we derive $\lambda_{C}(n) \leq \frac{c_{\Delta} W}{\Theta(n) r(n)}$ w.h.p.. Since $r(n)$ is at least $\Theta(\sqrt{\log n} / \sqrt{n})$ to ensure connectivity [1], and throughput is defined as a high probability quantity, we have $\lambda_{C}(n) \leq c_{\Delta} W / \Theta(\sqrt{n \log n})$.

Theorem 2: The $2 D$ throughput benefit ratio is upper bounded by a constant: $\alpha(n)=\Theta(1)$.

Proof: Gupta\&Kumar [1] already establishes a lower bound for the throughput of flow schemes, $\lambda_{F}(n) \geq$ $\Theta\left(\frac{c_{1} W}{(1+\Delta)^{2} \sqrt{n \log n}}\right)$ where $c_{1}>0$ is a constant. Combined with Theorem 1, we get $\alpha(n)=\lambda_{C}(n) / \lambda_{F}(n) \leq \Theta(1)$. Meanwhile, since the set of coding schemes includes all flow schemes, $\alpha(n) \geq 1$, thus $\alpha(n)=\Theta(1)$.

Observe that the constant throughput benefit ratio holds for a random network deployed in any arbitrarily shaped region. First, the upper bound for the throughput of the coding scheme still holds. Second, the constructive lower bound of Gupta\&Kumar [1] can in fact be extended to arbitrarily shaped regions, even though the asymmetry may cause the constructive scheme in [1] to have a skewed load distribution for some cut. Since the region is of fixed finite area, the throughput loss due to the asymmetric shape will be a fixed constant factor as $n$ increases. Thus, we have shown that network coding combined with wireless broadcast provides no order-different improvement on the throughput of a random network deployed in any arbitrarily shaped $2 D$ region.

\section{Bounds on the Throughput Benefit Ratio $\alpha$ in the Protocol Model}

Our technique for bounding the constant $\alpha$ is similar to those we used in Subsection IV-B. We will first provide a tighter upper bound for the capacity of the sparsity cut and thus the coding scheme throughput $\lambda_{C}(n)$; then, we construct a tighter lower bound for the flow scheme throughput $\lambda_{F}(n)$.

Define the two way cut capacity as the maximum possible number of bits that can cross the cut concurrently, regardless of direction. Note that an upper bound for the two way cut capacity is automatically an upper bound for the one way cut capacity.

Henceforth, the notation $A B$ will refer to the line segment between $A$ and $B$, where $A$ and $B$ are points in the network deployment space, and $|A B|$ refers to the distance between $A$ and $B$.
Lemma 2: The two way capacity of a cut $\Gamma$ for a $2 D$ region is upper bounded by $W\left(\frac{l_{\Gamma}}{\Delta r(n)}+1\right)$

Proof: See Appendix A.

Theorem 3: The throughput of the coding scheme on a $2 D$ square random network is upper bounded by

$$
\lambda_{C}(n) \leq \frac{2 W}{n}\left(\frac{1}{\Delta r(n)}+1\right) .
$$

Proof: The proof is almost the same as that of Theorem 1 , except that now we use the tighter bound for cut capacity in Lemma 2, and we prove this by showing that for any given constant $\epsilon>0, \lambda_{C}(n) \leq \frac{2 W}{n(1-\epsilon)}\left(\frac{1}{\Delta r(n)}+1\right)$ for large $n$.

Theorem 4: The throughput of the flow scheme on a $2 D$ square random network is lower bounded by

$$
\lambda_{F}(n) \geq \frac{W}{c_{\Delta 4} \sqrt{\pi}(1+\Delta) n r(n)}
$$

where $c_{\Delta 4}=\max \left\{2, \sqrt{\Delta^{2}+2 \Delta}\right\}$.

We use a similar technique as [24] that divides the area into deterministic squarelets, but [24]'s focus is an alternative proof of Gupta \&Kumar's capacity order result [1] while we try to bound the constant factor here.

Proof: We show that, for any given constant $\epsilon>0$, $\lambda_{F}(n) \geq \frac{W}{c_{\Delta 4} \sqrt{\pi}(1+\Delta)(1+\epsilon) n r(n)}$ for large $n$.

For the case of $\Delta \leq 1$, we choose a larger transmission radius $r^{\prime}(n)=\frac{\sqrt{\pi}}{\sqrt{\Delta^{2}+2 \Delta}} r(n)$ than the $r(n)$ required for connectivity. We then divide the square region into square cells of size $\sqrt{\pi} r(n) \times \sqrt{\pi} r(n)$. By the same union bound argument as in [25], with high probability each square cell contains at least one node.

Locate the $X$ axis on one edge of the square, and the $Y$ axis on the edge perpendicular to the first. We construct a scheme that is a natural extension of the $1 D$ scheme described in detail in [15] and [21]. Set the transmission radius to $r^{\prime}(n)$, and route along cells that lie on a line parallel to either the $X$ axis or $Y$ axis. The scheduling and routing along parallel lines to the axis has a phase for scheduling data routed to a cell header node that can be randomly chosen in each cell and a phase for scheduling routing between cell headers. The scheduling is evenly distributed along the line. See [15] and [21].

To deal with the interference between the parallel lines of routing, we do time division multiplexing between the lines. Time is slotted and assign half of the time to transmitting data along lines parallel to $X$, and the rest of the time to transmitting data along lines parallel to $Y$. In addition, only transmissions along every other line are scheduled at a given time, while the other lines remain silent. Thus, each line is scheduled to transmit every 4 time slots. Data is routed parallel to the $X$ axis until it reaches a cell that is in the same $Y$ line as the destination cell; then, it starts to route along a line parallel to the $Y$ axis.

We can show by a Chernoff bound and union bound, the load among lines is asymptotically balanced (equally loaded); more specifically, with high probability the ratios of the loads between all pairs of lines is arbitrarily close to 1 for large $n$. Then, using analogous results from the 1-D case [15], [21], we evaluate the cut capacity utilization level and derive the lower bound, $\lambda_{F}(n) \geq \frac{W}{2 \sqrt{\pi}(1+\Delta)(1+\epsilon) n r(n)}$ for large $n$. 
For the case $\Delta>1$, we choose $r^{\prime}(n)=\sqrt{\pi} r(n)$. Again, time is slotted and now each line is scheduled to transmit every $2 \sqrt{\Delta^{2}+2 \Delta}$ time slots lines instead of every two lines. Thus a throughput of $\frac{W}{\sqrt{\Delta^{2}+2 \Delta} \sqrt{\pi}(1+\Delta)(1+\epsilon) n r(n)}$ is achievable for this case. Combining these two cases proves the theorem.

Theorem 5: The throughput improvement of the flow scheme on a $2 D$ square random network is upper bounded by

$$
\alpha(n) \leq 2 c_{\Delta 4} \sqrt{\pi} \frac{(1+\Delta)}{\Delta}
$$

for large $n$, where $c_{\Delta 4}=\max \left\{2, \sqrt{\Delta^{2}+2 \Delta}\right\}$

Proof: This result follows from Theorems 3 and 4.

\section{Throughput Order Improvement of the Coding Scheme in the Physical Model}

Denote the coding scheme and flow scheme throughputs under the physical model as $\lambda_{C}^{p}(n)$ and $\lambda_{F}^{p}(n)$ correspondingly. We assume $\beta>1$ in (1) which is true for most wireless communication systems. Under the physical model, receivers could lie in a line vertical to the cut line. However, there are still certain geometric properties that the transmissions need to satisfy. We have the following lemma, which is actually also true under the protocol model.

Lemma 3: Under both the physical and protocol models, for any two transmissions across a cut, $S_{1} \rightarrow R_{1}$ and $S_{2} \rightarrow$ $R_{2}{ }^{1}$, the line segments $S_{1} R_{1}$ and $S_{2} R_{2}$ have no intersection point and $S_{1} S_{2}$ cannot be perpendicular to the cut line.

Proof: The proof is similar to that of Lemma 5. Now we connect $S_{1} S_{2}$, and draw the perpendicular bisector $h$ of $S_{1} S_{2}$. For any communication model, protocol or physical, $\left|S_{1} R_{1}\right|<\left|S_{2} R_{1}\right|,\left|S_{2} R_{2}\right|<\left|S_{1} R_{2}\right|$ is always true. Thus $S_{1}$ and $R_{1}$ lie on one side of $h$, and $S_{2}$ and $R_{2}$ lie on the opposite side. So $S_{1} R_{1}$ and $S_{2} R_{2}$ can never intersect (this is in general true for any two transmissions, not necessarily two across a cut). Now, if $S_{1} S_{2}$ is perpendicular to the cut line, $h$ is parallel to the cut line. Then, since $S_{1}$ and $R_{1}$ lie on one side of $h$ and $S_{2}$ and $R_{2}$ the other, one of the transmissions would not cross the cut. Thus, $S_{1} S_{2}$ cannot be perpendicular to the cut line.

Theorem 6: The coding throughput of a $2 D$ random network under the physical model is upper bounded by

$$
\lambda_{C}^{p}(n) \leq \Theta(W / \sqrt{n})
$$

for large $n$.

Proof: As shown in [1], (1) implies that

$$
r_{k, j} \geq(1+\Delta) r_{i, j}
$$

for all $k \in K$, where $K$ is the node set of all other nodes that are simultaneously transmitting and $\Delta=\beta^{\frac{1}{\gamma}}-1$. Since $\beta>1$, we always have $\Delta>0$.

Under the physical model, nodes can transmit with any hop distance $r$ so long as the signal to noise ratio is satisfied. We order the transmissions across the cut in one direction in the same way as in Lemma 6. Now, senders are on one side of the cut line since we focus on the one way cut capacity. Applying

\footnotetext{
${ }^{1}$ Note that each transmission could have multiple receivers, we just pick any one of them.
}

Lemma 3 and (2), we argue in a similar way as Lemma 2. We have $S_{j}(x)-S_{j-1}(x) \geq \Delta \min \left\{r_{j}, r_{j-1}\right\}$ for $j=2, \ldots, m$ where $m$ is the total number of transmissions across the cut in one direction and $r_{j}=\left|S_{j} R_{j}\right|$. The sparsity cut line has unit length; thus, we have

$$
\sum_{j=2}^{m} \min \left\{r_{j}, r_{j-1}\right\} \leq 1 / \Delta .
$$

Consider a band region of size $\frac{2}{\sqrt{n}} \times|A B|$ with the cut line in the center; by a Chernoff bound argument, we know that with high probability the number of nodes in this region is less than or equal to $3 \sqrt{n}$; thus, there are at most $3 \sqrt{n}$ transmissions with a radius less than $\frac{1}{\sqrt{n}}$ across the cut. Then there are at least $m-9 \sqrt{n}$ transmissions crossing the cut such that any one of them has $S_{j^{\prime}} R_{j^{\prime}}$ satisfying $\min \left\{r_{j^{\prime}}, r_{j^{\prime}-1}, r_{j^{\prime}+1}\right\} \geq \frac{1}{\sqrt{n}}$. Then, by (3), we have

$$
(m-9 \sqrt{n}) \frac{1}{\sqrt{n}} \leq \frac{1}{\Delta} \Rightarrow m \leq \Theta(\sqrt{n}) .
$$

Thus, we obtain an order upper bound for the sparsity cut capacity, and then derive an upper bound for the coding throughput in the same way as we did for the protocol model in Theorem 3.

Theorem 7: (Franceschetti et al. [26]) For any wireless medium with $\gamma>2$, the flow throughput of a $2 D$ physical random network is lower bounded by

$$
\lambda_{F}^{p}(n) \geq \Theta(W / \sqrt{n})
$$

for large $n$.

Theorem 8: For any wireless medium with $\gamma>2$, the throughput benefit ratio of a $2 D$ physical random network is a constant, and $\lambda_{F}^{p}(n)=\lambda_{C}^{p}(n)=\Theta(W / \sqrt{n})$.

Proof: This follows from Theorems 6 and 7.

Thus, we have established that coding schemes provide no more than a constant factor throughput improvement over flow schemes for the physical communication model.

\section{Throughput Benefit Bound for Multi-Source Multicast}

For flow schemes, [27] shows the capacity for single session multicast and [28] shows the multicast capacity for multiple source multicast. To our knowledge, there has been no study on the benefit of network coding on multiple source multicast in wireless networks.

We use the same random deployment model as in previous sections and focus on the protocol communication model. There are $n_{s}$ sources randomly chosen from the $n$ nodes, and each is a source for $n_{d}$ randomly chosen destination nodes. We still use $\lambda_{F}(n)$ and $\lambda_{C}(n)$ to denote the flow and coding throughput per session, respectively, with the modification that, in the multicast case, each session's throughput is the data rate to each of the $n_{d}$ receivers from the source.

First we study the case when $n_{s}=n$ and $n_{d}=\Theta(1)$, and we show that network coding provides at most a constant factor gain in this case. Then we study the case when $n_{s}=n^{\epsilon}$ and $n_{d}=n^{1-\epsilon}$ and upper bound the network coding benefit ratio for both $1 D$ and $2 D$ networks.

The following theorem that bounds the coding gain in multicast networks is a direct corollary of Theorem 1. 
Theorem 9: The multicast coding gain of a random network with $n$ sources each with $\Theta(1)$ random destinations is upper bounded by a constant.

Proof: The cut technique employed in the unicast case is still valid. So Theorem 1 holds. In addition, if we treat each session's $\Theta(1)$ receivers as $\Theta(1)$ independent unicast sessions from the source, then a lower bound of the same order is established for the throughput of the flow scheme from Gupta\&Kumar's result. Thus, when $n_{d}=\Theta(1)$, we have $\alpha(n)=\Theta(1)$.

Now we consider the case when $n_{s}=n^{\epsilon}$ and $n_{d}=n^{1-\epsilon}$, as in [28]. We consider two cases here: $1 D$ and $2 D$ networks. The $1 D$ deployment makes the routing combinatorics much simpler and we are able to derive tight bounds on the throughput and, thus, coding benefit ratio.

Theorem 10: The multicast coding gain of a $1 D$ random network with $n^{\epsilon}$ sources each with $n^{1-\epsilon}$ random destinations is upper bounded by $\frac{1+\Delta}{1+\Delta / 2}$.

The proof applies the same argument as in Theorem 5 and 6 of [15], with the only difference being that, instead of having roughly $n / 4$ sources that need to cross the sparsity cut, now w.h.p. we will have close to $n^{\epsilon} / 2$ sources that need to cross the sparsity cut. The reason is that as $n$ become large, the $n^{1-\epsilon}$ receivers for each source guarantees that each source must send traffic across the sparsity cut. Thus, with the same argument as in Theorems 5 and 6 of [15], we obtain $\lambda_{F}(n)=$ $\frac{W}{(1+\Delta) n^{\epsilon}}, \lambda_{C}(n)=\frac{W}{(1+\Delta / 2) n^{\epsilon}}$ and $\alpha(n)=\frac{1+\Delta}{1+\Delta / 2}$.

For the $2 D$ networks, we use two techniques to upper bound the coding throughput. First notice that Lemma 1 still holds. Applying the same proof as in Theorem 1 with $\Theta\left(n^{\epsilon}\right)$ sources that need to cross the cut, we have

$$
\lambda_{C}(n) \leq \Theta\left(\frac{W}{n^{\epsilon} r(n)}\right)
$$

The second technique bounds the coding benefit ratio through an information distance metric.

Lemma 4: The network coding benefit ratio, $\alpha(n)$, for a multicast random wireless network with $n_{s}=n^{\epsilon}, n_{d}=n^{1-\epsilon}$ is $\alpha(n)=O(r(n) \sqrt{n} \sqrt{\log n})$

Proof: We need a lowerbound on $\lambda_{F}(n)$ and an upperbound on $\lambda_{C}(n)$. It has been shown in [28] that

$$
\lambda_{F}(n)=\Theta\left(\frac{1}{\sqrt{n^{\epsilon} \log n}}\right)
$$

Thus we focus on $\lambda_{C}(n)$.

Any coding scheme disseminates data from $n_{s}$ mutually independent sources to the $n_{s} n_{d}$ destinations. With high probability, each bit of data must traverse a distance that is at least $L\left(n_{d}\right)=\Theta\left(\sqrt{n_{d}}\right)$. Although established in the context of flow schemes, [28], it holds more generally for any coding scheme. The potential benefit of network coding is that one transmission can potentially deliver information to more than one node. In particular, one coding transmission potentially delivers information to $O\left(n \pi r^{2}(n)\right)$ nodes (whp), where the distance to a neighbor is at most $r(n)$. Thus each transmission contributes $O\left(n r^{3}(n)\right)$ bit.meters (whp). As the total number of simultaneous transmissions is upperbounded by $1 /\left(\pi r^{2}(n)\right)$, the total number of bit.meters accumulated in one transmission interval is $O(n r(n))$ (whp). Now, $\lambda_{C}(n)$, the coding scheme throughput, equals the ratio of number of bit.meters produced per session per unit time divided by the number of meters that a bit must traverse. Thus

$$
\lambda_{C}(n)=\frac{O(n r(n))}{n_{s} L\left(n_{d}\right)}=\frac{O(n r(n))}{n_{s} \Theta\left(\sqrt{n_{d}}\right)}=O\left(r(n) \sqrt{n^{1-\epsilon}}\right)
$$

This coupled with the expression for $\lambda_{F}(n)$ above yields the desired result.

Theorem 11: The network coding throughput gain is upper bounded by $O\left(\sqrt{\log n \sqrt{n^{1-\epsilon}}}\right)$ for randomly deployed $2 D$ wireless networks.

Proof: [28] shows that the flow throughput is $\Theta\left(\frac{W}{\sqrt{n^{\epsilon} \log n}}\right)$. Combined with (4), this yields an upper bound on the coding scheme benefit ratio, $\alpha(n)=O\left(\frac{\sqrt{\log n}}{\sqrt{n^{\epsilon} r(n)}}\right)$. On the other hand Lemma 4 provides another upper bound, $\alpha(n)=O(r(n) \sqrt{n} \sqrt{\log n})$. Thus,

$$
\alpha(n)=\min \left\{O\left(\frac{\sqrt{\log n}}{\sqrt{n^{\epsilon}} r(n)}\right), O(r(n) \sqrt{n} \sqrt{\log n})\right\} .
$$

The order of this upper bound is maximized when $r(n)=$ $\Theta\left(n^{-\frac{1+\epsilon}{4}}\right)$. Si nce the coding benefit ratio is the maximum coding benefit over all possible $r(n)$ s, we app ly $r(n)=$ $\Theta\left(n^{-\frac{1+\epsilon}{4}}\right)$ to (5) and get an upper bound for the coding throughput benefit ratio as $O\left(\sqrt{\log n \sqrt{n^{1-\epsilon}}}\right)$.

\section{Throughrut Benefit Bound For ARbitrary NODE DEPLOYMENTS}

We begin by first reviewing results from wireline networks that will be employed below. A wireline network can be represented by a graph, and the min cut of the graph induces an upper bound on the throughput. Given an arbitrary directed graph, Leighton and Rao [29] show that, the max flow for several variations of the multicommodity flow problem on a wireline network is within an $O(\log n)$ factor of the upper bound implied by the min-cut, where $n$ is the total number of nodes in the network. We first review the result in [29] in the context of our application, state the necessary assumptions, and then state and prove our result.

Consider a directed graph $G(V, E)$, where $V$ is the vertex set and $E$ is the edge set. There is a capacity $c_{e}$ associated with each edge $e \in E$. Let $S \subseteq V$ denote a set of vertices generating demands and assume equal demands $D(u, v)=1$ (without loss of generality) for all $u, v \in S$, $u \neq v$. Define a $c u t$ to be $(U, \bar{U})$ where $\bar{U}=V \backslash U \neq \emptyset$. Let $\langle U, \bar{U}\rangle=\left\{\left(v_{i}, v_{j}\right) \mid v_{i} \in U, v_{j} \in \bar{U}\right\}$. The cut capacity is defined as $C(U, \bar{U})=\sum_{e \in\langle U, \bar{U}\rangle} c_{e}$. Let $D(U, \bar{U})=$ $\sum_{u \in U \cap S} \sum_{v \in \bar{U} \cap S} 1$ denote the aggregate traffic demand between sources in $U$ and destinations in $\bar{U}$. We use the term sparsity to denote $C(U, \bar{U}) / D(U, \bar{U})$. Define the minimum sparsity $\xi$ of $G$ as

$$
\xi=\min _{U \subset V} \frac{C(U, \bar{U})}{D(U, \bar{U})},
$$

and, as before, a sparsity cut $\Gamma_{s}$ refers to a cut that has the minimum sparsity. A consequence of Theorem 17 in [29] is that the maximum flow for the multicommodity flow problem is within a $\log n$ factor of the sparsity cut where $n$ denotes the size of $S$. 
We now focus on a wireless network. Let $V^{\prime}$ denote the set of nodes, $S^{\prime}$ denote the set of source/destinations with cardinality $n$. Let $\Upsilon_{C}$ denote a specific network coding scheme that determines when nodes are allowed to transmit, what data is coded and decoded, and what routes data takes. Define a node set $m$ as $v$ 's neighbor receiver set if nodes in $m$ are all the sucessful receivers of some broadcast transmissions from $v$ under $\Upsilon_{C}$. Let $N(v)$ denote the set of all neighbor receiver sets that node $v \in V^{\prime}$ has successfully broadcast data to under $\Upsilon_{C}, m \in N(v)$. Let $\left\{A_{u, v}(t)\right\}$ denote the arrival process for source $u$ and destination $v, u, v \in V^{\prime}$, i.e., $A_{u, v}(t)$ denotes the number of bits that arrive at $u$ destined for $v$ in the interval $[0, t)$. Last, let $R_{u, v}(t)$ denote the number of bits decoded by $v \in V^{\prime}$ that originated from $u \in V^{\prime}$. We make the following assumptions regarding $\Upsilon_{C}$ and $\left\{A_{u, v}(t)\right\}$ :

1) Long term per session arrival rates exist and without loss of generality, are equal to one,

$$
\lim _{t \rightarrow \infty} \frac{A_{u, v}(t)}{t}=1, \quad \forall u, v \in S^{\prime}
$$

2) Network conservation of flow, in other words

$$
\lim _{t \rightarrow \infty} \frac{R_{u, v}(t)}{t}=1, \quad \forall u, v \in S^{\prime}
$$

3) The long-term data rate broadcasted from a node to each of its neighbor receiver sets exists. In particular, let $b_{u, m}(t)$, denote the total amount of data delivered by broadcast transmissions from $u$ to the neighbor receiver set $m \in N(u)$ by time $t$ under $\Upsilon_{C}$.

$$
c_{u, m}=\lim _{t \rightarrow \infty} \frac{b_{u, m}(t)}{t}, \quad \forall u \in V^{\prime} ; m \in N(u)
$$

Theorem 12: Whenever a network coded network satisfies the assumptions above, there exists a flow-based network that can achieve source destination throughputs within a factor of $\log n$ of those of the network coded network.

Proof: We first map the coding network into a directed graph, and then apply the Leighton/Rao result to prove the theorem.

Consider coding scheme $\Upsilon_{C}$. Focus on node $u \in V^{\prime}$. For each neighbor receiver set $m \in N(u)$ such that $c_{u, m}>0$, create a virtual node $v_{u, m}$ and add a directed edge $\left(u, v_{u, m}\right)$ with capacity $c_{u, m}$ and infinite capacity edges $\left(v_{u, m}, v\right), \forall v \in$ $m$.

Denote the resulting directed graph $G_{0}=\left(V_{0}, E_{0}\right)$ and take the same set of sources and destinations to be $S^{\prime}$. Given that the flow rate out of the network equals the flow rate in, it follows that the minimum sparsity, $\xi \geq 1$. By Theorem 17 of [29], there exists a multicommodity flow routing solution $\Upsilon_{F}$ that achieves per session rates of $\Omega(\xi / \log n)=\Omega(1 / \log n)$. This solution determines how flow is routed though the network. The method used to construct the directed graph based on $\left\{c_{u, m}: m \subseteq N(u) ; u \in V^{\prime}\right\}$ guarantees the existence of schedules at all nodes to support this routing scheme.

Note that if we change the traffic demand assumption to be symmetric only, i.e., $D(u, v)=D(v, u)$, then based on the $O\left(\log ^{3} n\right)$ max-flow min-cut result established in [30], [31] and following the exact same proof above, we show that the flow based scheme can achieve throughputs within a factor of $\log ^{3} n$ of those of the network coding scheme.

\section{CONCLUSIONS}

As summarized in Table I, under the random network framework of [1], we have shown that the network coding and broadcasting benefit is upper bounded by a constant in the multiple unicast scenario for both the protocol model and the physical model, and we have bounded this constant for the protocol model. The bounds on the constant are still loose, and we suspect that the constant factor is quite a bit smaller; specifically, we conjecture it is 2 , and proving it even partially may involve solving the well-known Li\&Li conjecture [6], which is still open. The reason for conjecture that the gain is 2 is because network coding can only potentially improve the outgoing information rate from a node, while the incoming information rate is still constrained as previously, and the information flow conservation law requires a balance between outgoing and ingesting rate at a node. Bounds on the benefit of network coding and broadcasting in the multiple source multicast case and for the multiple unicast case in an arbitrary network have also been presented.

Our work, combined with the previous work of [6], is negative about network coding's utility for improving network capacity. However, in general, we believe that what we have learned is not that network coding is not helpful, but rather better guidance on where network coding's true advantage lies. We feel the key utility of network coding lies in its ability to blur the information's identities. For communication in lossy, unstable, dynamic environments (e.g. Delay/Disruption Tolerant Networks (DTN)), distributed storage/recoveries in disaster, fault-tolerant situations (e.g. growth codes [33]) etc., network coding's ability to blur the information identities can help to balance out the risks and redistribute them uniformly across all packets, which could benefit the delay, reliability and robustness of the system. If we think about the capacity benefit of network coding on directed graphs, the benefit essentially also comes from its ability to blur the information identity. For our case of bidirectional wireless networks modeled as using lossless communication channels, even though network coding is able to blur the information identities, the overall information content cannot be compressed with network coding and thus the capacity gain for this case is limited.

\section{APPENDIX}

\section{A. Proof of Lemma 2}

Lemma 5: For any two transmissions across a cut, $S_{1} \rightarrow$ $R_{1}$ and $S_{2} \rightarrow R_{2}^{2}$, the line segments $S_{1} R_{1}$ and $S_{2} R_{2}$ do not intersect and $R_{1} R_{2}$ cannot be perpendicular to the cut line.

Proof: As shown in Fig. 6, draw the perpendicular bisector $h$ of $R_{1} R_{2}$. By the protocol communication model, we know $\left|S_{1} R_{1}\right| \leq\left|S_{1} R_{2}\right|$ and $\left|S_{2} R_{2}\right| \leq\left|S_{2} R_{1}\right|$. Thus $S_{1}$ and $R_{1}$ lie on one side of $h$ and $S_{2}$ and $R_{2}$ lie on the opposite side. Hence $S_{1} R_{1}$ and $S_{2} R_{2}$ cannot intersect. If $R_{1} R_{2}$ is perpendicular to the cut line, as we see in Fig. 6, then $h$ is parallel to the cut line. Therefore, one of the senders must lie at the same side of the cut line as the receiver, contradicting the assumption of the lemma. Thus, $R_{1} R_{2}$ cannot be perpendicular to the cut line.

\footnotetext{
${ }^{2}$ Note that each transmission could have multiple receivers, we just pick any one of them.
} 
TABLE I

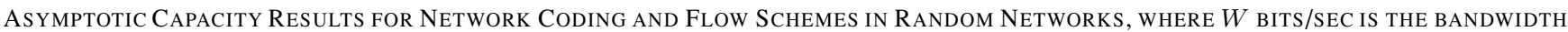
LIMIT FOR EACH TRANSMISSION, $\Delta$ IS A CONSTANT THAT DEPENDS ON THE PROPERTIES OF THE WIRELESS MEDIUM, $c_{\Delta 4}=\max \left\{2, \sqrt{\Delta^{2}+2 \Delta}\right\}$, $c_{\Delta_{3}}=\min \left\{\frac{192}{\pi \Delta^{3}}, \frac{256}{\sqrt{3} \pi \Delta^{2}}\right\}$.

\begin{tabular}{|c|c|c|c|c|}
\hline \multicolumn{2}{|l|}{ Model } & Flow Throughput $\lambda_{F}(n)$ & Coding Throughput $\lambda_{C}(n)$ & Benefit ratio $\alpha(n)=\frac{\lambda_{C}(n)}{\lambda_{F}(n)}$ \\
\hline Unicast Protocol Model & $\begin{array}{l}1 D \\
2 D \\
3 D \\
k D\end{array}$ & $\begin{array}{c}\frac{\frac{2 W}{(1+\Delta) n}}{c_{\Delta 4} \sqrt{\pi}(1+\Delta) n r(n)} \\
\Theta\left(\frac{W}{(1+\Delta)^{2} \sqrt{n \log n}}\right)[32] \\
\Theta\left(\frac{W}{\sqrt[k]{n \log k-1 n}}\right)\end{array}$ & $\begin{array}{c}\leq \frac{2 W}{(1+\Delta / 2) n} \\
\frac{2 W}{n(1-\epsilon)}\left(\frac{1}{\Delta r(n)}+1\right) \\
\leq \frac{c_{3} W}{n r(n)^{2}} \\
\Theta\left(\frac{\frac{W}{\sqrt[k]{n \log k-1 n}}}{\sqrt{n} \log ^{2}}\right)\end{array}$ & $\begin{array}{c}\frac{1+\Delta}{1+\Delta / 2} \\
\leq 2 c_{\Delta 4} \sqrt{\pi} \frac{1+\Delta}{\Delta} \\
\Theta(1) \\
\Theta(1)\end{array}$ \\
\hline Unicast Physical Model & $\begin{array}{l}1 D \\
2 D\end{array}$ & $\begin{array}{c}\Theta\left(\frac{W}{n}\right) \\
\Theta\left(\frac{W}{\sqrt{n}}\right)[26]\end{array}$ & $\begin{array}{l}\Theta\left(\frac{W}{n}\right) \\
\Theta\left(\frac{W}{\sqrt{n}}\right)\end{array}$ & $\begin{array}{l}\Theta(1) \\
\Theta(1)\end{array}$ \\
\hline Multicast Protocol Model & $\begin{array}{l}1 D \\
2 D\end{array}$ & $\begin{array}{c}\frac{W}{(1+\Delta) n^{\epsilon}} \\
\Theta\left(\frac{W \sqrt{n^{1-\epsilon}}}{\sqrt{n \log n}}\right)\end{array}$ & $\begin{array}{c}\frac{W}{(1+\Delta / 2) n^{\epsilon}} \\
\leq \min \left\{O\left(\frac{W}{n^{\epsilon} r(n)}\right), O\left(W \sqrt{n^{1-\epsilon}} r(n)\right)\right\}\end{array}$ & $O\left(\sqrt{\frac{1+\Delta}{\log n \sqrt{n^{1-\epsilon}}}}\right)$ \\
\hline
\end{tabular}

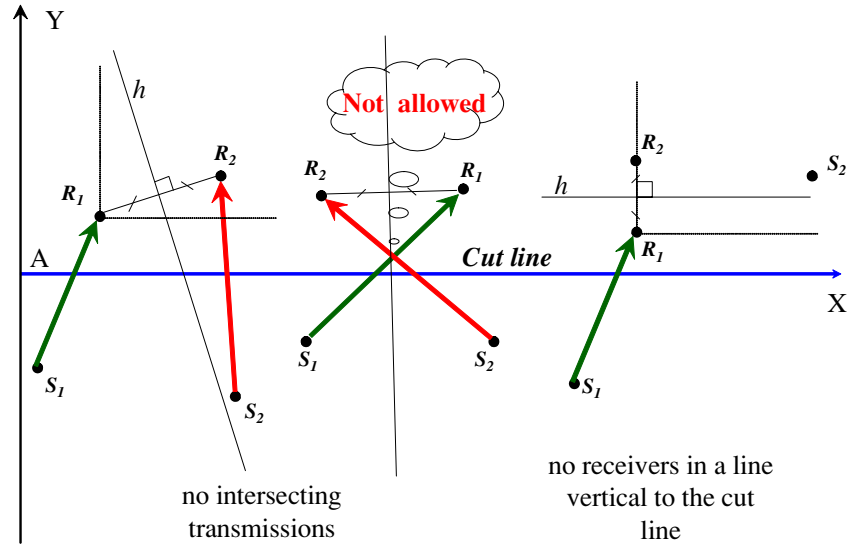

Fig. 6. Geometric properties of transmissions across a cut

Next, we construct a coordinate system for a cut $A B$. Let $A$ be the origin, the line of $A B$ be the $X$ axis, and a line perpendicular to $A B$ be the $Y$ axis (see Fig. 7). We denote the $X$ coordinate of a node $R$ by $R(x)$. Order all of the simultaneous transmissions across the cut by their intersecting points with the $X$ axis (the cut line), from small $X$ coordinates to large ones. Label the sender-receiver pairs of the ordered transmissions as $S_{1} \rightarrow R_{1}, S_{2} \rightarrow R_{2}, \ldots, S_{m} \rightarrow R_{m}$, where $m$ is the total number of scheduled simultaneous transmissions across the cut. Denote $X_{k}=\max \left\{S_{k}(x), R_{k}(x)\right\}$ for $1 \leq k \leq m$ and $X_{0}=0$.

Lemma 6: $X_{k}-X_{k-1} \geq \Delta r(n)$ for all $2 \leq k \leq m$.

Proof: W.l.o.g., consider the first two consecutive transmissions in the ordered list $S_{1} \rightarrow R_{1}$ and $S_{2} \rightarrow R_{2}$. By Lemma 5, we know $R_{2}(x)>R_{1}(x)$; otherwise the ordering should be switched.

Scenario 1: We first consider scenario 1 as shown in Fig. 7 , where the senders are on one side of the cut and the receivers are on the other. Fig. 7 shows the case for $S_{1}(x) \leq R_{1}(x)$. There are two possibilities for $R_{2}(y)$ : either $R_{2}(y) \leq R_{1}(y)$ or $R_{2}(y)>R_{1}(y)$.

When $R_{2}(y) \leq R_{1}(y)$, from the facts that $\left|S_{1} R_{2}\right| \geq(1+$ $\Delta) r(n),\left|S_{1} R_{1}\right| \leq r(n), S_{1}(x) \leq R_{1}(x)$ and $S_{1}(y)<R_{1}(y)$ we easily get $R_{2}(x)-R_{1}(x)>\Delta r(n)$; thus, $X_{2}-X_{1}>$ $\Delta r(n)$.

When $R_{2}(y)>R_{1}(y)$ as shown in Fig. 7, we look at the tri-

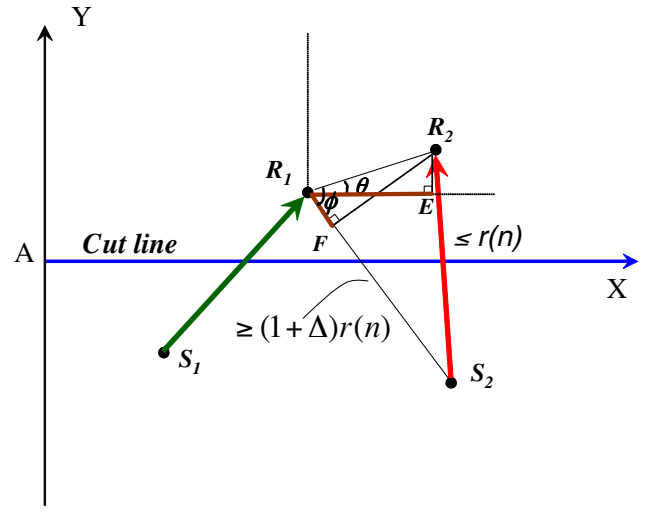

Fig. 7. Tighter bound for packing transmissions across a cut: Scenario 1

angle $R_{1} R_{2} S_{2}$. By the protocol model, $\left|R_{1} S_{2}\right| \geq(1+\Delta) r(n)$, $\left|R_{2} S_{2}\right| \leq r(n)$. From $R_{2}$, drawing $R_{2} F \perp R_{1} S_{2}$ where $F$ resides on $R_{1} S_{2}$, we have $\left|R_{1} F\right| \geq\left|R_{1} S_{2}\right|-\left|R_{2} S_{2}\right| \geq \Delta r(n)$. Note that $F$ has to lie between $R_{1}$ and $S_{2}$, since $\phi<90^{\circ}$ (because $\left.\left|R_{1} S_{2}\right|>\left|R_{2} S_{2}\right|\right)$. Now, from $R_{1}$ draw a line $R_{1} E$ parallel to the $X$ axis, and from $R_{2}$ draw $R_{2} E$ perpendicular to $R_{1} E$ and intersecting with $R_{1} E$ at $E$. It is easy to see that $\left|R_{1} E\right|=R_{2}(x)-R_{1}(x)$. Since $S_{2}(y)<R_{1}(y), \theta<\phi$. Thus $\left|R_{1} E\right|>\left|R_{1} F\right|$; then, $R_{2}(x)-R_{1}(x)>\Delta r(n)$ and $X_{2}-X_{1}>\Delta r(n)$.

Combining these two cases, yields $X_{2}-X_{1}>\Delta r(n)$ for the situation when $S_{1}(x) \leq R_{1}(x)$. A similar argument applies when $S_{1}(x)>R_{1}(x)$.

Scenario 2: The other scenario is when the two senders are on different sides of the cut. This scenario divides into two cases.

Case a): When $S_{2}(y)>R_{1}(y)$, as shown in Fig. 8, using a similar argument we can show $\left|R_{1} E\right|>\left|R_{1} F\right|>\Delta r(n)$; thus, $X_{2}-X_{1}>\Delta r(n)$.

Case b): Fig. 9 shows the other case, when $S_{2}(y) \leq$ $R_{1}(y)$. We deduce from the figure that $S_{2}(x)-R_{1}(x) \geq$ $\sqrt{\Delta^{2}+2 \Delta} r(n)>\Delta r(n)$.

By the axis symmetry of the cut line and sender-receiver symmetry we have $X_{2}-X_{1}>\Delta r(n)$ for this scenario.

Combining Scenario 1 and Scenario 2, and noting that $S_{1} R_{1}$ and $S_{2} R_{2}$ are an arbitrary adjacent pair of transmissions anywhere in the ordered list $\left\{\cdots, S_{k} R_{k}, \cdots, 1 \leq k \leq m\right\}$, 


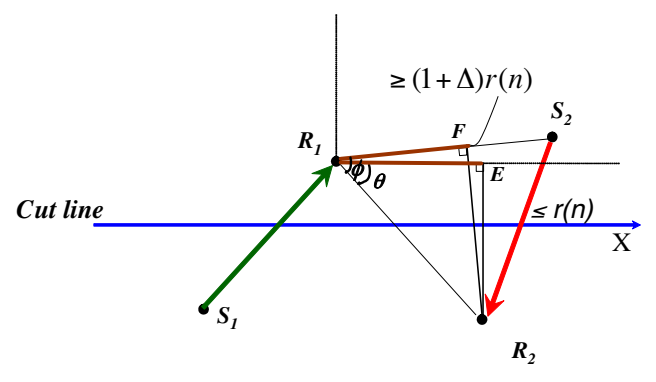

Fig. 8. Tighter bound for packing transmissions across a cut: Scenario 2 case a

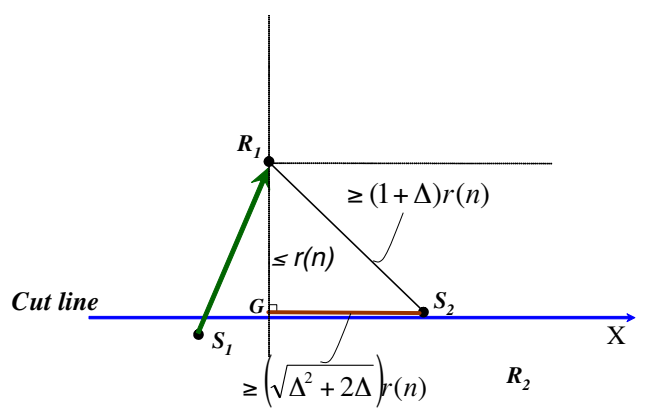

Fig. 9. Tighter bound for packing transmissions across a cut: Scenario 2 case b)

the Lemma is proved.

Applying Lemma 6 to the fact that $\sum_{1 \leq k \leq m}\left(X_{k}-X_{k-1}\right) \leq$ $l_{\Gamma}$ yields Lemma 2.

\section{REFERENCES}

[1] P. Gupta and P. R. Kumar, "The capacity of wireless networks," IEEE Transactions On Information Theory, vol. 46, pp. 388-404, Mar. 2000.

[2] M. Grossglauser and D. Tse, "Mobility increases the capacity of ad-hoc wireless networks," in INFOCOM, 2001.

[3] O. L. A. Ozgur and D. Tse, "Hierarchical cooperation achieves optimal capacity scaling in ad hoc networks," IEEE Transactions on Information Theory, vol. 53, pp. 3549-3572, October 2007.

[4] B. Liu, P. Brass, O. Dousse, P. Nain, and D. Towsley, "Mobility improves coverage of sensor network," in ACM MobiHoc, 2005.

[5] R. Ahlswede, N. Cai, S. Li, and R. Yeung, "Network information flow," IEEE Transactions on Information Theory, vol. 46, pp. 1204-1216, July 2000.

[6] Z. Li and B. Li, "Network coding in undirected networks," in CISS, 2004.

[7] S. Deb, M. Effros, T. Ho, D. R. Karger, R. Koetter, D. S. Lun, M. Medard, and N. Ratnakar, "Network coding for wireless applications: A brief tutorial," in IWWAN, 2005.

[8] D. S. Lun, M. Medard, and R. Koetter, "Efficient operation of wireless packet networks using network coding," in IWCT, 2005.

[9] D. S. Lun, M. Medard, and R. Koetter, "Network coding for efficient wireless unicast," in IEEE International Zurich Seminar on Communications, 2006.

[10] S. Katti, D. Katabi, W. Hu, and R. Hariharan, "The importance of being opportunistic: Practical network coding for wireless environments," in Allerton, 2005.

[11] R. Alimi, L. Li, R. Ramjee, H. Viswanathan, and Y. Yang, "ipack: innetwork packet mixing for high throughput wireless mesh networks," in INFOCOM, 2008

[12] J. Le, J. C. S. Lui, and D. M. Chiu, "How many packets can we encode? - an analysis of practical wireless network coding," in INFOCOM, 2008.

[13] A. Keshavarz-Haddad and R. Riedi, "Bounds on the benefit of network coding: Throughput and energy saving in wireless networks," in INFOCOM, 2008.

[14] J. Liu, D. Goeckel, and D. Towsley, "The throughput order of ad hoc networks employing network coding and broadcasting," in MILCOM, 2006.

[15] J. Liu, D. Goeckel, and D. Towsley, "Bounds on the gain of network coding and broadcasting in wireless networks," in INFOCOM, 2007.
[16] J. J. Garcia-Luna-Aceves, H. R. Sadjadpour, and Z. Wang, "Challenges: towards truly scalable ad hoc networks," in MobiCom, 2007.

[17] J. J. Garcia-Luna-Aceves, H. R. Sadjadpour, and Z. Wang, "Extending the capacity of ad hoc networks beyond network coding," in IWCMC, 2007.

[18] J. J. Garcia-Luna-Aceves, H. R. Sadjadpour, and Z. Wang, "The capacity and energy efficiency of wireless ad hoc networks with multi-packet reception," in MobiHoc, 2008.

[19] K. Lu, S. Fu, and Y. Qian, "Capacity of random wireless networks: Impact of physical-layer network coding," in ICC, 2008.

[20] C. Chen and H. Xiang, "The throughput order of ad hoc networks with physical-layer network coding and analog network coding," in ICC, 2008.

[21] J. Liu, On Joint Coding and Combinatorial Optimization in Communication Networks. University of Massachusetts Amherst, 2008.

[22] R. Motwani and P. Raghavan, Randomized algorithms. Cambridge University Press, 1995.

[23] T. Cover and J. Thomas, Elements of Information Theory. Wiley Series in Telecommunications, New York, NY, USA: John Wiley \& Sons, 1991.

[24] S. Kulkarni and P. Viswanath, "A deterministic approach to throughput scaling in wireless networks," IEEE Transactions On Information Theory, vol. 50, no. 6, pp. 1041-1049, 2004.

[25] P. Kumar, "A correction to the proof of a lemma in "the capacity of wireless networks'," IEEE Transactions on Information Theory, vol. 49, p. 3117, November 2003.

[26] M. Franceschetti, O. Dousse, D. Tse, and P. Thiran, "On the throughput capacity of random wireless networks," IEEE Transactions on Information Theory, 2006.

[27] P. Jacquet and G. Rodolakis, "Multicast scaling properties in massively dense ad hoc networks," in ICPADS, 2005.

[28] S. Shakkottai, X. Liu, and R. Srikant, "The multicast capacity of large multihop wireless networks," in MobiHoc, 2007.

[29] T. Leighton and S. Rao, "Multicommodity max-flow min-cut theorems and their use in designing approximation algorithms," Journal of ACM vol. 46, no. 6, pp. 787-832, 1999.

[30] G. Even, J. Naor, B. Schieber, and M. Sudan, "Approximating minimum feedback sets and multicuts in directed graphs," Algorithmica, vol. 20, pp. 151-174, 1998.

[31] P. Klein, S. Plotkin, S. Rao, and E. Tardos, "Bounds on the max-flow min-cut ratio for directed multicommodity flows," Algorithmica, vol. 22, pp. 241-269, 1997.

[32] P. Gupta and P. Kumar, "Internets in the sky: The capacity of three dimensional wireless networks," 2001.

[33] A. Kamra, J. Feldman, V. Misra, and D. Rubenstein, "Growth codes: Maximizing sensor network data persistence," in SIGCOMM, 2006.

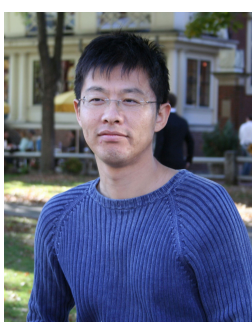

Junning Liu received his B.E. in Engineering Physics and M.S. in Pattern Recognition and Intelligent Systems both from Tsinghua University, China. $\mathrm{He}$ received his Ph.D. degree in Computer Science from University of Massachusetts at Amherst in 2008. He is currently a research engineer at youtube, Google.

His research interests include joint coding and combinatorial optimization, network coding, wireless networks, collaborative filtering and personalized recommendation algorithms. 


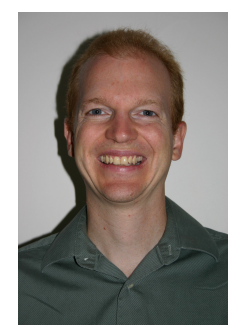

Dennis Goeckel split time between Purdue University and Sundstrand Corporation from 1987-1992, receiving his BSEE from Purdue in 1992. From 1992-1996, he was a National Science Foundation Graduate Fellow and then Rackham Pre-Doctoral Fellow at the University of Michigan, where he received his MSEE in 1993 and his Ph.D. in 1996, both in Electrical Engineering with a specialty in Communication Systems. In September 1996, he joined the Electrical and Computer Engineering department at the University of Massachusetts, where he is currently a Professor. His current research interests are in the areas of communication systems and wireless network theory.

Dr. Goeckel was the recipient of a 1999 CAREER Award from the National Science Foundation for "Coded Modulation for High-Speed Wireless Communications". He was a Lilly Teaching Fellow at UMass-Amherst for the 2000-2001 academic year and received the University of Massachusetts Distinguished Teaching Award in 2007. He served as an Editor for the IEEE Journal on Selected Areas in Communications: Wireless Communication Series during its transition to the IEEE Transactions on Wireless Communications from 1999-2002, a Technical Program Committee Co-Chair for the Communication Theory Symposium at IEEE Globecom 2004, and a Technical Program Committee Co-Chair for the Wireless Communications Symposium at IEEE GlobeCom 2008. He is currently an Editor for the IEEE Transactions on Communications.
Don Towsley holds a B.A. in Physics (1971) and a Ph.D. in Computer Science (1975) from University of Texas. He is currently a Distinguished Professor at the University of Massachusetts in the Department of Computer Science.He has held visiting positions at IBM T.J. Watson Research Center, Yorktown Heights, NY; Laboratoire MASI, Paris, France; INRIA, SophiaAntipolis, France; AT\&T Labs - Research, Florham Park, NJ; and Microsoft Research Lab, Cambridge, UK. His research interests include networks and performance evaluation. He currently serves as Editor-in-Chief of IEEE/ACM Transactions on Networking, and on the editorial boards of Journal of the ACM and IEEE Journal on Selected Areas in Communications and has previously served on numerous other editorial boards. He was Program Cochair of the joint ACM SIGMETRICS and PERFORMANCE'92 conference, the Performance 2002 conference, and INFOCOM'09. He is a member of ACM and ORSA. He has received the 2007 IEEE Koji Kobayashi Award, the 2007 ACM SIGMETRICS Achievement Award,the 2008 ACM SIGCOMM Lifetime Achievement Award, a 2008 SIGCOMM Test-of-Time Paper Award, the 1998 IEEE Communications Society William Bennett Best Paper Award, and numerous conference/workshop best paper awards. Last, he has been elected Fellow of both the ACM and IEEE. 